\title{
Enhancement of Integrated Power System Analysis Package Capability by Integration of Object-Oriented Physical System Modeling Language
}

\author{
Komsan Hongesombut Member (R\&D Center, Tokyo Electric Power Co.) \\ Tsuyoshi Takazawa Member (R\&D Center, Tokyo Electric Power Co.) \\ Yasuyuki Tada Member (R\&D Center, Tokyo Electric Power Co.) \\ Yasunori Mitani Member (Kyushu Institute of Technology)
}

Keywords: object-oriented physical system modeling, power system modeling, power system simulation, power system analysis, graphical user interface

The increase in size and complexity of interconnected electric power systems, coupled with the industrial commitment to maximize security at a minimum cost, has led to the development of many special software packages for power system analysis. These tools are used by power system operators and system planning engineers to ensure that the electric power system is able to operate without instability under a wide range of system conditions. This paper presents a new development of an integrated power system analysis package named MidFielder, which is now integrated with a recent technology using object-oriented physical system modeling language for very flexible studies in power systems. To authors' knowledge, it is the first attempt to include this technology into the power system analysis package.

We have feedback from users both in industrial and academic sectors that not only should the simulation packages provide computational efficiency, user-friendly environment, but also should have the flexibility and ability of easy modeling. This is very important for a rapid way for research and development in power systems. Many commercial power system analysis packages available on the market provide the possibility to model controllers such as governors and exciters, but do not allow the user to simulate generic models of generators or networks. Therefore, it is cumbersome for research purposes. On the other hand, if this problem can be solved, power system analysis package will have the completeness for industrial, educational and research purposes.

Recently, development within the field of object-oriented physical system modeling is a fast-growing area of modeling and simulation that provides a computer-supported way of doing mathematical and equation-based modeling. Modelica is the object-oriented simulation language being developed to advance the state of system simulation technology. This technology has revolutionized the way in which the model can be constructed similar to the physical system; therefore, a straightforward modeling is enabled. The motivation for introducing Modelica in Tokyo Electric Power Company (TEPCO) project is to remove some of the necessary skills in computer programming and numerical analysis, so that power system engineers are able to focus on describing the model instead of the actual lowlevel implementation. The development of power system models of appropriate fidelity is a key aspect of power system simulation processes. The models must allow all relevant multi-disciplinary modeling criteria, e.g. model structure and data handling, to be computed efficiently, easily, and with sufficient accuracy. Recently, the project team at TEPCO is attempting to create an interface system of an integrated power system analysis package with a software tool using object-oriented physical modeling language Modelica to aid the power system engineers in the rapid generation and evaluation of feasible model design. This involves managing a large set of power system data connected by various types of constraints.

Recently, ObjectStab is only one freely available Modelica library. In ObjectStab, power system topology and parameter data are entered using a graphical user interface. While it has a powerful graphical editor for composing models, however, this advantage is turned into a disadvantage for large power system problems. Power system network configuration is very difficult to be entered manually, e.g. 10,000-bus power system. Therefore, it limits the capability of users to study large power system problems. In addition, ObjectStab is created as a tool for educational purpose. There are limited numbers of industrial grade models. In order to gain accuracy and reliability in the models, we developed MidFielderAddin library. It has matching accuracy of models with MidFielder and includes various models in commercial power system analysis packages such as PSS/E.

Our vision for the MidFielder integrated Modelica environment is to fulfill essentially all the requirements for a general purposes integrated power system analysis package combined with the specific needs for modeling and simulation environments. We believe that our purposed concept will have a great impact for the development of future integrated power system analysis package so that the integrated power system analysis package will have the completeness for industrial, educational and research purposes.

This paper is organized as follows. Firstly, the MidFielder environment and its main features are described in section 2. Section 3 describes details of the developed parts for the construction of MidFielder integrated Modelica. Section 4 presents two topologies which can be used to enhance the capability of MidFielder for studies in model comparisons. Section 5 shows how to implement MidFielder integrated Modelica to power system studies. An example of how to model a user-defined generator is given. Single machine and multimachine study cases are used to verify the compatibility of the developed Modelica library used in this project with MidFielder and other power system analysis packages. The results are compared among different power system analysis packages. Section 6 follows with a summary. 


\title{
Enhancement of Integrated Power System Analysis Package Capability by Integration of Object-Oriented Physical System Modeling Language
}

\author{
Komsan Hongesombut* Member \\ Tsuyoshi Takazawa* Member \\ Yasuyuki Tada* Member \\ Yasunori Mitani** Member
}

\begin{abstract}
There are many commercial power system analysis packages available on the market. Although most of these tools are typically computationally efficient, they do not provide the flexibility and ability to simulate generic models of generators or networks. This is cumbersome for research and development purposes. The development of power system models of appropriate fidelity is a key aspect of power system simulation processes. The models must allow all relevant multi-disciplinary modeling criteria, e.g. model structure and data handling, to be computed efficiently, easily, and with sufficient accuracy. This paper presents how the adoption of recent technology on object-oriented physical systems modeling can be implemented with an integrated power system analysis package MidFielder. Used in combination with MidFielder, this can provide the completeness of power system analysis package for industrial, educational and research purposes. In order to realize the proposed interface system, this paper also discusses about methods to manage a large set of power system data by using database technology and means of graphical user interface (GUI).
\end{abstract}

Keywords: object-oriented physical system modeling, power system modeling, power system simulation, power system analysis, graphical user interface

\section{Introduction}

The increase in size and complexity of interconnected electric power systems, coupled with the industrial commitment to maximize security at a minimum cost, has led to the development of many special software packages for power system analysis. These tools are used by power system operators and system planning engineers to ensure that the electric power system is able to operate without instability under a wide range of system conditions. This paper presents a new development of an integrated power system analysis package named MidFielder, which is now integrated with a recent technology using object-oriented physical system modeling language for very flexible studies in power systems. To authors' knowledge, it is the first attempt to include this technology into the power system analysis package.

We have feedback from users both in industrial and academic sectors that not only should the simulation packages provide computational efficiency, user-friendly environment, but also should have the flexibility and ability of easy modeling. This is very important for a rapid way for research and development in power systems. Many commercial power system analysis packages available on the market provide the possibility to model controllers such as governors and

\footnotetext{
* Power System Technology Group, R\&D Center, Tokyo Electric Power Co.

4-1, Egasaki-cho, Tsurumi-ku, Yokohama 230-8510

** Department of Electrical Engineering, Kyushu Institute of Technology

1-1, Sensui-cho, Tobata-ku, Kitakyushu 840-8550
}

exciters, but do not allow users to simulate generic models of generators or networks. Therefore, it is cumbersome for research purposes. On the other hand, if this problem can be solved, power system analysis packages will have the completeness for industrial, educational and research purposes.

Recently, development within the field of object-oriented physical system modeling is a fast-growing area of modeling and simulation that provides a computer-supported way of doing mathematical and equation-based modeling. Modelica is the object-oriented simulation language being developed to advance the state of system simulation technology ${ }^{(1)(2)}$. This technology has revolutionized the way in which the model can be constructed similar to the physical system; therefore, a straightforward modeling is enabled. The motivation for introducing Modelica in Tokyo Electric Power Company (TEPCO) projects is to remove some of the necessary skills in computer programming and numerical analysis, so that power system engineers are able to focus on describing the model instead of the actual low-level implementation. The development of power system models of appropriate fidelity is a key aspect of power system simulation processes. The models must allow all relevant multi-disciplinary modeling criteria, e.g. model structure and data handling, to be computed efficiently, easily, and with sufficient accuracy. Recently, the project team at TEPCO is attempting to create an interface system of an integrated power system analysis package with a software tool using object-oriented physical modeling language Modelica to aid the power system engineers in the rapid generation and evaluation of feasible model 
design. This involves managing a large set of power system data connected by various types of constraints.

Recently, ObjectStab is only one freely available Modelica library ${ }^{(3)(4)}$. Studies using this library are shown in these literatures ${ }^{(5)-(10)}$. In ObjectStab, power system topology and parameter data are entered using a graphical user interface (GUI). While it has a powerful graphical editor for composing models, however, this advantage is turned into a disadvantage for large-scale power system problems. Power system network configuration is very difficult to be entered manually, e.g. 10,000-bus power system. Therefore, it limits the capability of users to study large-scale power system problems. In addition, ObjectStab is created as a tool for educational purpose. There are limited numbers of industrial grade models. In order to gain accuracy and reliability in the models, we developed MidFielderAddin library (previously, known as IMPACT to Modelica package). It has matching accuracy of models with MidFielder and includes various models in commercial power system analysis packages such as in PSS/E.

This paper is organized as follows. Firstly, the MidFielder environment and its main features are described in section 2 . Section 3 describes details of the developed parts for the construction of MidFielder integrated Modelica. Section 4 presents two topologies which can be used to enhance the capability of MidFielder for studies in model comparisons. Section 5 shows examples on how to implement MidFielder integrated Modelica to power system studies. An example of how to model a user-defined generator is given. Single machine and multimachine study cases are used to verify the compatibility of the developed Modelica library used in this project with MidFielder and other power system analysis packages. The results are compared among different power system analysis packages. Section 6 follows with a summary.

\section{MidFielder Environment and its Main Fea- tures}

The basic structure of MidFielder environment is illustrated schematically in Fig. 1. Generally, MidFielder is divided into six layers, one concerned with database (DB), one with DB handling, one with data reading script, one with conventional text file, one with application and one with post process. Note that the highlighted blocks in Fig. 1 are additionally developed components in MidFielder. However, this information should be given on how object-oriented physical modeling language Modelica can be integrated into MidFielder environment. Each layer includes.

(1) DB layer MidFielder supports single-user and multi-database servers. These enable users to access to the same project database via user accounting and project access rules. MidFielder controls access to a number of databases. Databases are storage areas used by the server to partition information. For example, a typical installation may have a database for as-built power system used for power system operation and control purpose. Used to keep all information about power system data for a planning stage, it may also have a planning database. Used for training and testing purposes, it may have private databases used by individuals to store personal information. Because power system network grows and changes continually, additions and changes to database structures or schemes are a constant part of development process. MidFielder uses GUI-based tools, to package and integrate with web server, database server and various tools onto the Windows environment. Managing the creation of database objects such as tables, stored procedure, and views by using GUI-based tools offers a powerful and rapid

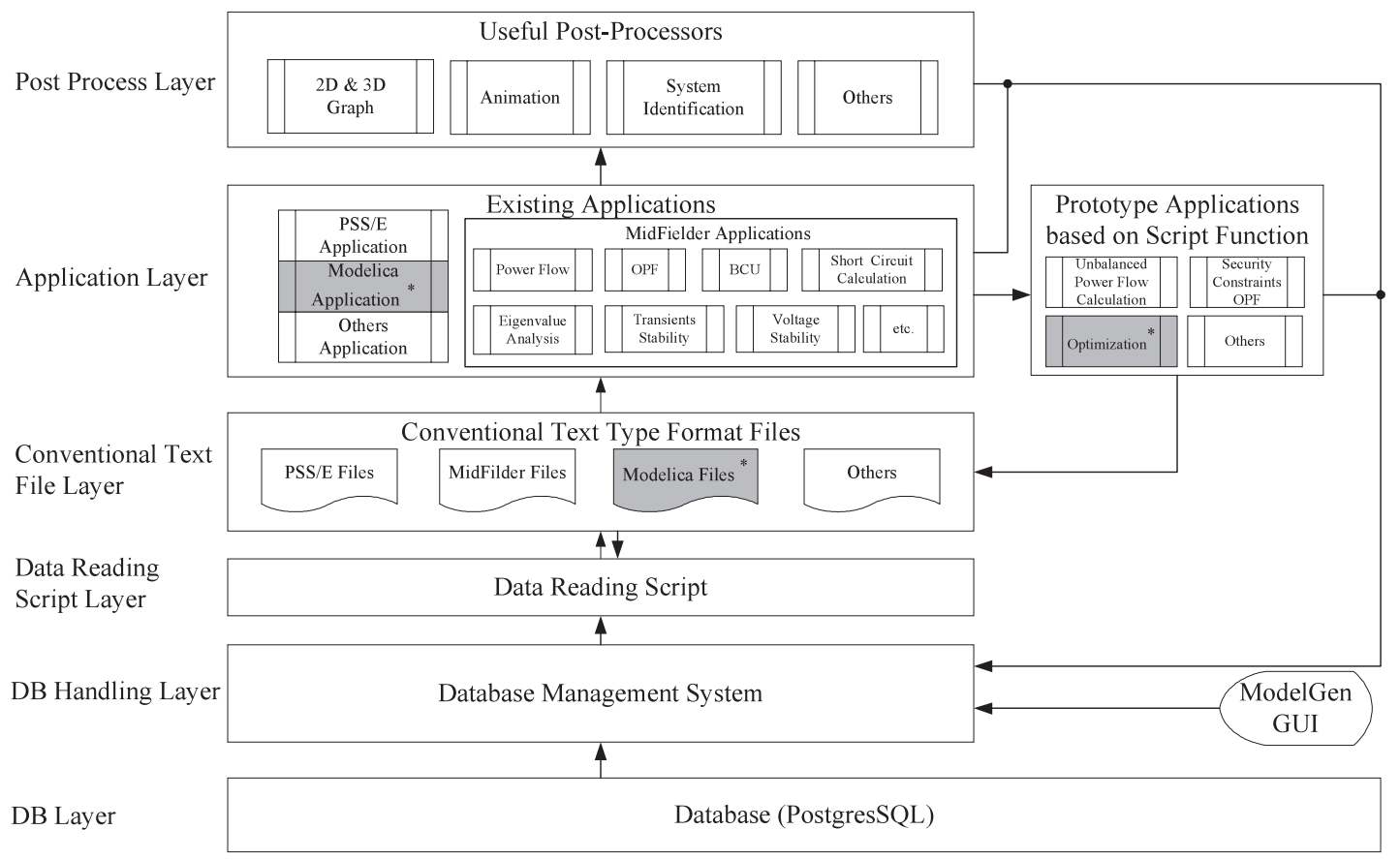

— Data and communication path

Fig. 1. MidFielder environment and its main features 
way to automate the details of the work. By using a general database for GUI, it is possible to analyze large power systems and to construct a new integrated package of tools containing with another system like SCADA.

(2) DB handling layer The DB handling layer is responsible for the management of power system data. In order to minimize data redundancy and increase efficiency in terms of execution speed, computational resources and maintainability, MidFielder comes with a powerful data management system. MidFielder is structured on different understanding of storing data. Generally, all power system data are organized around networks according to their base case definitions, e.g. the common data of as-built power systems of utilities or data of same set used many times. The definition may include operational data and resulting data of several scenarios. The incremental information for defining power systems can be added in the data structure. Therefore, it is effective to handle several alternative study cases at the same time, which is useful in the power system planning stage. All information data is non-redundant and the change of any data will automatically be updated to the most recent version. This method allows the fastest possible program handling since users do not require to perform time consuming procedures like editing, saving or updating data by themselves. In addition, data handling of large power system is very complicated on conventional text-based format because management of data ID such a node ID is as human jobs. In the MidFielder environment, all power system analytical data is inputted by the useful ModelGen interface.

(3) Data reading script layer Data reading script layer is a common gateway interface that serves data from a variety of software formats such as Y-method, PSS/E, Modelica, etc. This provides the fastest way to migrate old power system projects of other applications to MidFielder with less efforts and without breaking the original project data. The main purpose of using this layer is to remove some of the necessary skills in application data handling because inputting power data directly in text-based format is fairly laborious. Currently, MidFielder provides standard translators for conversion of PSS/E and Y-Method data into MidFielder data format. In this research, we developed an additional translator which enables automatic conversion of data formats into Modelica.

(4) Conventional text file layer This temporary layer serves as the feeder to application programs. Basically, ModelGen provides an easy-to-use high level user interface that works quite well for inputting power system data. In addition to purely graphical inputting of power system data using the ModelGen, MidFielder also provides a text-based environment for building power systems. For certain cases, there is the possibility that users do not prepare power system data using the GUI in ModelGen, but already have text files in another data format. For these cases, users may quickly start running old power system projects in MidFielder from this layer immediately. At this step, MidFielder uses conventional text files as inputs for appropriate translators to change data from one format to another. If MidFielder is run in the automatic mode, these generated conventional text files can be ignored and are not necessary to be accessed, but are useful for debugging the errors. Furthermore, for certain applications, users may use these output conventional text files to run in PSS/E, Y-method or Modelica directly. By this way, MidFielder becomes a key data manager that helps users improve data organization and time in project development, integration and migration.

(5) Application layer Application layer can be classified into two types; existing applications and prototype applications based on script functions. Existing applications consist of a set of well-tested programs that enable the efficient simulation of a wide variety of applications. There are two types for existing applications; the ones belonging to TEPCO programs and the ones belonging to other's programs. As illustrated in Fig. 1, the TEPCO applications currently applied to MidFielder are as follows:

- APP (Advanced Power flow Program)

- VTS (Variable Time-step Simulation program)

- BCU (Boundary of stability-region based Controlling Unstable equilibrium point)

- LINEAR (Linearization and Eigenvalue Analysis Recipes)

- POPF (Powerful Optimal Power Flow program)

- SCC (Short-circuit Current Calculation program)

During the development process, if needs of studies using existing applications are not met, users can develop prototype applications that let users write a program with script functions. This allows unlimited applications to access to a common database in MidFielder. Here, prototype applications include unbalanced power flow calculation, security constraints OPF, optimization, etc. Details of each application and its related functions can be found in these references ${ }^{(11)-(14)}$.

(6) Post process layer The post-processor has been designed to allow users to have an easy access to the large amount of results produced by MidFielder applications. The post-processor module is very powerful and flexible. The user is able to plot the time histories of transient analyses, as well as the steady state response in the frequency domain. Overlaying of several plots is possible.

\section{The Proposed Method to Integrate Modelica into MidFielder Environment}

\subsection{Why Modelica in Our Research Tasks}

Modelica's strength as a modeling language for complex technical systems is well proven ${ }^{(1)(15)(16)}$. The modeling language Modelica has the following major advantages for our research tasks.

- It is an equation-based language that expresses relations and constraints in a declarative way, rather than forcing the programmer to handle control flow and data flow.

- Using acausal modeling as in Modelica, the network has not to be modeled using the traditional admittance matrix method and power system topology can be visualized.

- End users do not need to worry about implementation details of interface. The interface concept is formally defined in Modelica and allows, for example, two components with the same interface to be substituted. It is easy and natural to model new power system components, especially network models, as well as facilitates reuse of existing model components.

- In most cases, the performance of generated simulation 
code in Modelica is more efficient than straightforwardly written $\mathrm{C}$ code in many software packages, because additional opportunities for symbolic optimization are used by the system.

Due to these above benefits, the development and integration Modelica technology into integrated power system analysis package MidFielder can help us in many cases extensively cut the cost and time for model testing and development processes.

Here, we demonstrate how simple network models can be expressed using Modelica. The example below shows the use of such a blueprint TwoPin model class to define different parts of electrical network components. The TwoPin class shown in Fig. 2 has two pins, T1 and T2 that defines the voltage and current phasors.

It is now straightforward to create the impedance model by adding constitutive equations.

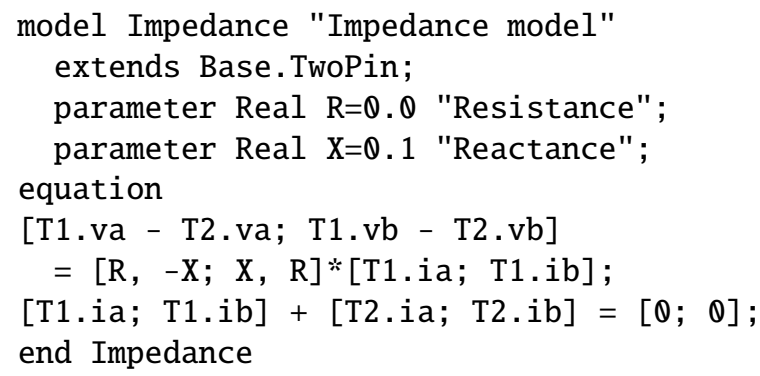

An Admittance model can also reuse TwoPin in a similar way by adding the constitutive equations for Admittance model.

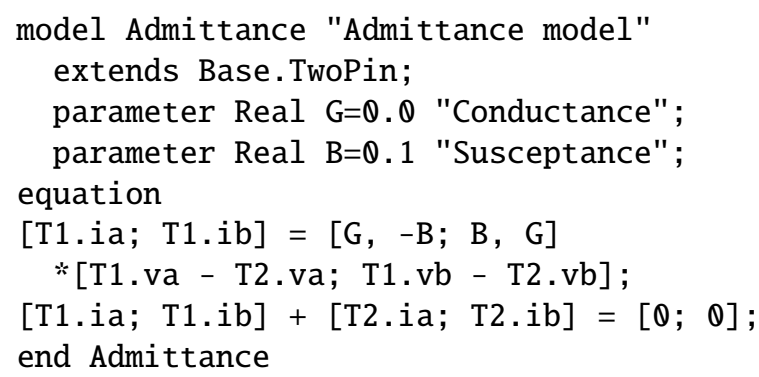

Having collected a small library of simple electrical components, we can now put all components together to construct the simple $\pi$ transmission line as shown in Fig. 3.

Connect equations are provided to connect the components in the circuit both inside and outside connectors.

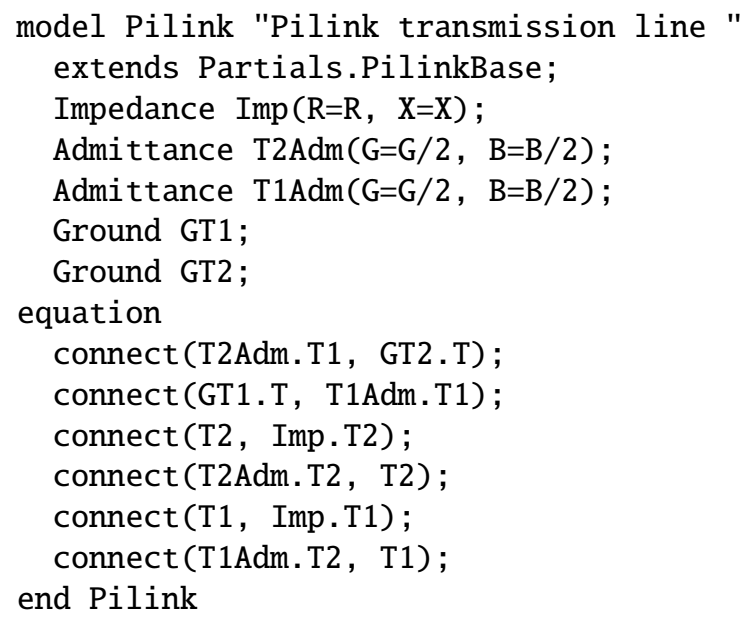

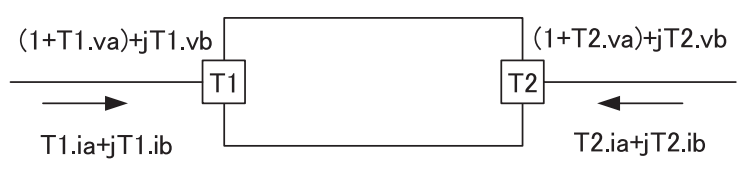

Fig. 2. TwoPin class that describes the general structure of electrical network components

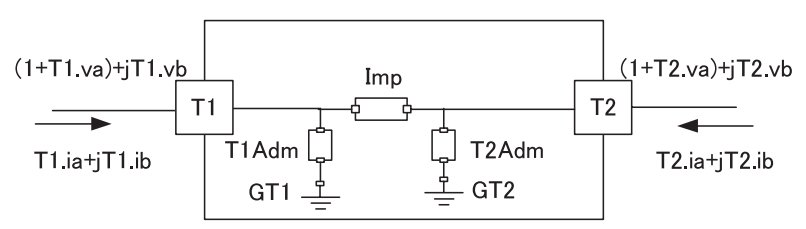

Fig. 3. $\pi$ transmission line model

\subsection{MidFielder Interface System with Modelica}

The proposed structure of interface system of MidFielder and Modelica is illustrated in Fig. 4. All power system analytical data is inputted in MidFielder environment using the functional ModelGen interface. ModelGen generates data for power system analysis using GUI and treats data of largescale power systems using a database technology. This function is very useful because large-scale power system data consist of several subsystems. As being the system that is able to input all system analytic data by an operation of GUI, users can make data comparatively easily and it is made practicable as a standard input interface of a large-scale power system data. The developed MidFielder to Modelica translator is capable of translating models in ModelGen into Modelica text-based format. There are three packages in the generated Modelica text file. One is used for loadflow calculation, namely Loadflow package. One contains the necessary dynamic and user-defined models, namely Models package. One is extended from the Loadflow package by replacing some dynamic models obtained from Models package, namely Dynamic package. In order to realize the proposed interface system, we have developed three main parts as follows:

(1) Communication of Modelica and MidFielder engine

Basically, MidFielder uses Python script to automate activities of standard MidFielder applications. Not only does this allow batch operation of MidFielder applications but is useful for solving optimization problems, if required. MidFielder employs a Python API to get applications to communicate with each other. Microsoft Windows applications use the DDE (Dynamic Data Exchange) protocol or ActiveX to exchange data and communicate with other Windows applications. This allows the automation of activities in MidFielder applications with other third-party applications. We developed a Modelica interface with MidFielder engine using DDE. DDE sends data between applications using Windows messages according to a documented protocol. A wellprogrammed client sends a Windows message to the server and carries on processing. Windows holds the message and sends it to the server when the server is ready to process it. DDE is straightforward to be implemented. All it can do is to transmit data. It can only control another application because 


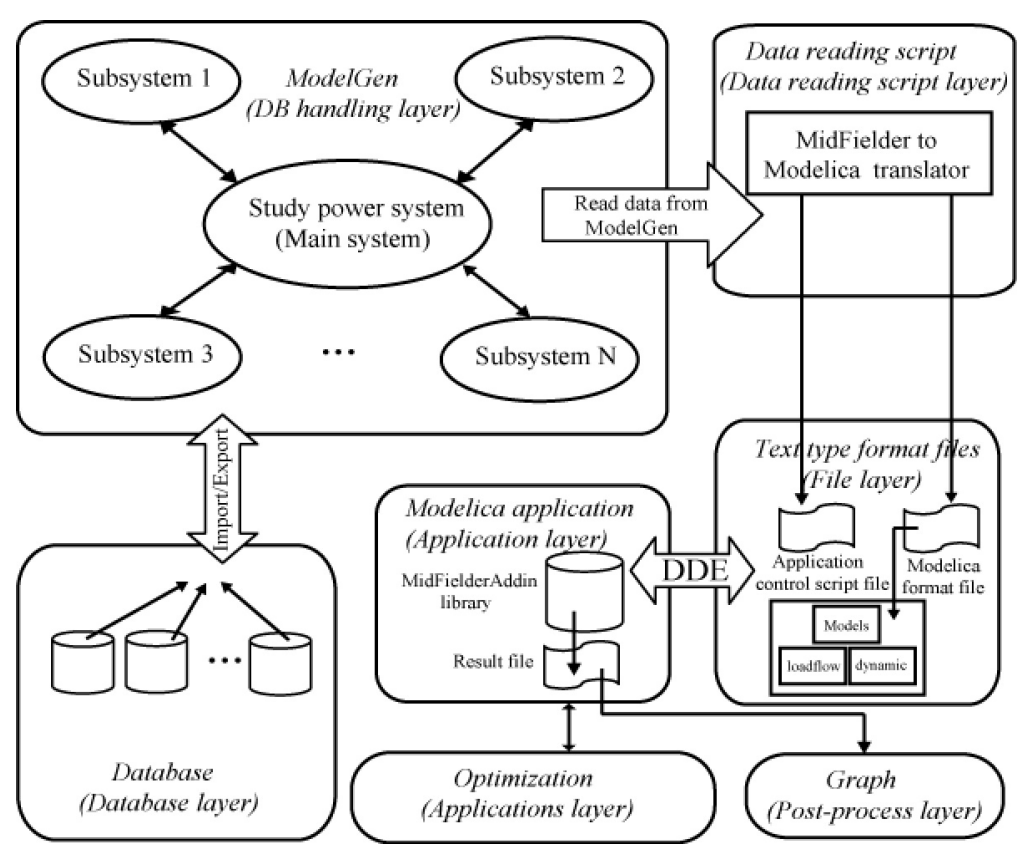

Fig. 4. Proposed MidFielder interface system with Modelica

the recipient can treat data as a command. For Modelica compiler, commercial software packages supporting Modelica language are now available, including Dymola ${ }^{(15)}$ and MathModelica ${ }^{(16)}$, which are amongst the leading objectoriented physical system modeling simulation tools available on current market. A free Modelica compiler is also available ${ }^{(17)}$. However, it has not fully supported all functions with our developed library. Therefore, Dymola has been chosen for use in this project ${ }^{(18)}$.

(2) MidFielderAddin library by Modelica language

MidFielderAddin library is used as a model template of MidFielder when the translator is calling. It was initially developed based on the inspiration of ObjectStab-A Modelica library for power system stability studies of M. Larsson ${ }^{(3)(4)}$. ObjectStab is good for use as a tool for educational purpose. It shows many successes in several literatures. However, there are numbers of differences that things have to be changed in this library to make models compatible with MidFielder such as the use of generator convention in expressing the stator voltage equation which is consistent with q-axis leading d-axis (d-axis leading q-axis for the original definition), the classification of models which can be composed of more devices, etc. Therefore, MidFielderAddin library does not need to come with ObjectStab. It can be considered as another generation of Modelica library for power system simulations. Additionally, in order to gain accuracy and reliability in the models, MidFielderAddin library has numbers of industrial grade models such as in PSS/E ${ }^{(19)}$. There is unlimited possibility when it comes to designing new network models. MidFielderAddin library presently contains these following models:

- Generators with constant frequency and voltage as slack or PV node or detailed generator models, i.e. D0QF0, D0Q0F1, D0Q1F1, D1Q1F1 and D1Q2F1, with excitation and governor control systems.

- Transmission lines in series impedance and $\pi$-link representation.

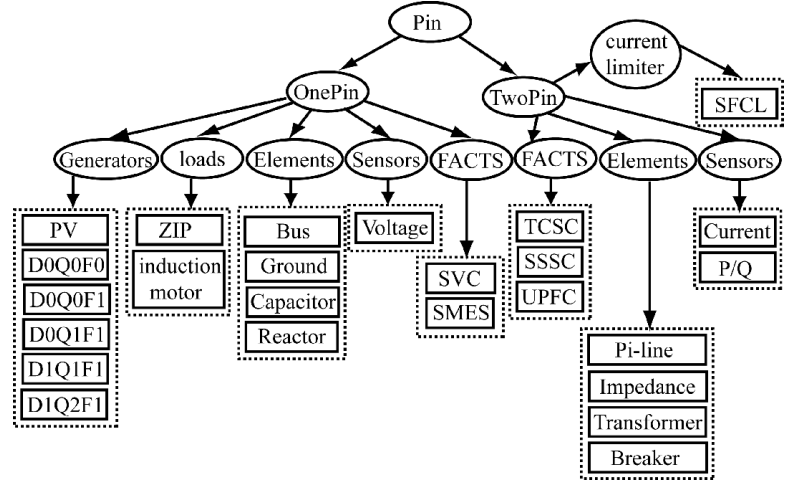

Fig. 5. Classification of standard model components in MidFielderAddin library

- Transformer with neglecting losses by magnetization and hysteresis currents.

- Static and dynamic loads.

- Buses.

- Faulted bus and faulted line with fault impedance.

- Measurement instruments of voltage, current, active and reactive powers.

- FACTS devices, i.e. Static Var Compensator (SVC), Thyristor Controlled Series Capacitor (TCSC), Superconducting Magnetic Energy Storage (SMES), Static Synchronous Series Compensator (SSSC), Static Synchronous Compensator (STATCOM), Unified Power Flow Controller (UPFC).

- Current limiter, i.e. Superconducting Fault Current Limiter (SFCL).

Figure 5 shows the classification of models in the developed MidFielderAddin library. Details of this library and examples for FACTS devices and SMES applications can be found in Ref. (20). Note that PSS/E models are additional models. As an example, we will explain how to model GENROU in PSS/E in section 5. 
(3) MidFielder to Modelica translator It is hard work for non-experience Modelica users to understand Modelica syntax and to input power system data in Modelica environment. Even experts, they may take much time to prepare all necessary power system data especially in large-scale power system problems. For this reason, we developed a translator to remove some of the necessary skills in Modelica programming ${ }^{(21)}$. With this translator, Modelica code can be generated conveniently with less efforts. Automatic conversion of data formats is very precise, detailed and reliable.

\section{Application in Model Comparison Studies}

The development MidFielder interface system with Modelica provides the possibility to study on power system models flexibly. Various models used in other commercial grade power system dynamics simulation tools or new user-defined models are easily investigated. One important application for electric utilities is dynamic data and simulation checking. This is not easy task and very time consuming procedure when large-scale power system problems are investigated by using traditional approaches. With our developed interface system, checks can be performed on different software packages using the same common database. This can aid in quickly detecting questionable parameters and help in assessing if power system models are realistically valid.

4.1 Danger of Model Comparisons We have noticed about the dangers of comparisons for model accuracy and efficiency using models on different simulation tools. The sources of errors which do appear in the process of evaluation are from many reasons such as accuracy of model parameters, the choice of which solver to use, human errors from data input, etc. The comparisons will be very hard because all of the errors are simultaneous and accumulating. How can we verify that the model is a good and reliable, and is valid for including in the simulations? To reduce the dangers, critical review on the assumptions, approximations and limitations behind the models on each software tool should be done and the results should be compared against the results from other software tools. This approach requires specialized knowledge about mechanism for handling and using tools in the model construction for specific applications and domains. In addition, it can be very hard and time consuming for modeling new power system components for every software tool, and sometimes we can hope only to get a partial answer to our questions. Therefore, the conclusions drawn from conventional model evaluation approaches are not only dependent on the properties of the investigated systems, but also on the software tools which are employed, as well as on how the power system components are modeled. Conclusions can differ when studying the same power system with different software tools.

\subsection{System Topologies for Model Comparison Studies}

The best way to verify the model is to compare to experimental results when this is possible. However, it is very difficult to have the actual system ready especially at future planning stage. We should perform sensitivity analysis of the model in simulation programs to ensure the validity of the model. If the simulation results are relatively insensitive to small variations of model parameters and simulation programs that are used, we will have strong reasons to believe

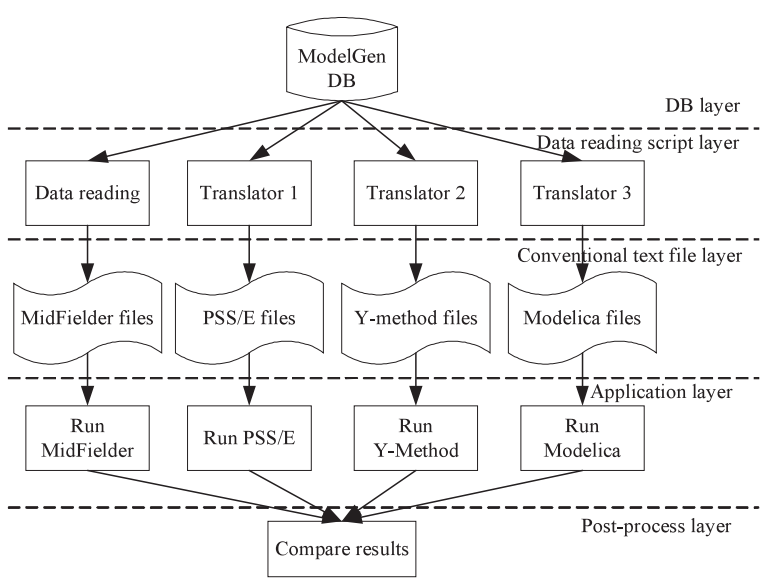

Fig. 6. Topology I

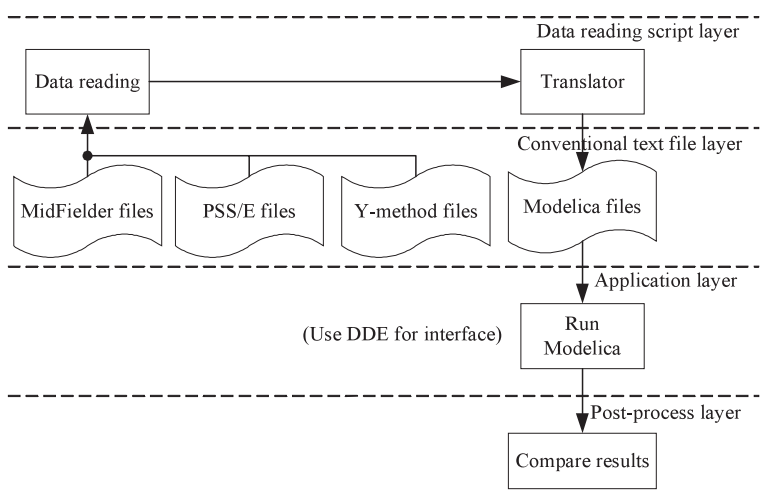

Fig. 7. Topology II

in the validity of the model. Before the model is going to be used in a project, to ensure that the model is highest precision possible, we should double check or triple check the model because the model could give us a false result. We propose the following two topologies in MidFielder for model comparison studies.

(1) Topology I The topology I is illustrated in Fig. 6. To reduce errors from human input, each simulation program utilizes a common database from ModelGen DB. The appropriate translator in data reading layer is applied so that power system data is automatically translated into appropriate data formats for each simulation program. Users may use the translated output files to run simulations of each simulation program manually. On the other hand, MidFielder uses DDE interface to start applications and automate processes of running and plotting.

Pros:

- Usually, the results obtained from commercial simulation programs are highly efficient in the computation and the results are reliable.

Cons:

- The sensitivity analysis of model parameters is difficult to be compared because all models are in different simulation environments.

- Each simulation program has some operational constraints and very often, the setup of study models can be setup only to make them very close, but not identical. In worse case situation, new network models cannot be 
modeled.

- While this method is straightforward for comparisons, it requires users who are widely skilled in handling each simulation program.

(2) Topology II The topology II is illustrated in Fig. 7. This topology employs equation-based model definition. The high-level of abstraction of equation-based models presents new challenges to modeling and simulation because equation-based model is useful in generating large number of test models quickly. This method directly converts data of MidFielder, PSS/E and Y-method from text- based format into Modelica file by using a translator script in data reading layer. Then all power system models are in the same environment in Modelica. MidFielder will use DDE interface to start Modelica compiler, and automate processes of application running and plotting.

\section{Pros:}

- User-defined models, both generator and network models, can be easily added meaning that if there is the difficulty in modeling in other simulation programs, all projects can be converted into Modelica by using the appropriate translator in data reading script layer. Then users can take advantages from Modelica for modeling.

- Power system engineers are able to focus on describing the model using equations, block diagrams or combined both ways. This provides the fastest way to evaluate new power system models in one efficient environment.

- This method allows efficient graph-theoretic algorithms to be applied in solving the equation set. In these, the equations may be sorted and symbolically rearranged, attempting to achieve a computationally feasible sequence. When one or more algebraic loops exist, the method for index reduction is used. In terms of simulation efficiency, the solver method in Modelica is typically faster than industrial grade tools such as PSS/E for small and medium power systems.

- The sensitivity analysis of model parameters is possible to be compared because all models are in the same Modelica environment.

Cons:

- Speed in simulation may be slow for very large-scale power system problems.

\section{Example Study Cases}

Our developed interface system and models in MidFielderAddin library were extensively tested. In this section, we briefly present some of example results, in order to illustrate the power of the Modelica mathematical modeling and simulation technology using our developed interface system.

5.1 Modeling Example of a User-Defined Generator (GENROU in PSS/E) The definition of connector used in MidFielderAddin library was already described in Ref. (20). Here, we are looking into only significant parts of the developed model. When representing GENROU in PSS/E by a block diagram ${ }^{(19)}$, the model is as Fig. 8. An important aspect of modeling with Modelica is the ability to construct models using an "acausal" formulation. This means that it is not necessary to decide in advance what the inputs and outputs of a model must be. This is a way that a model can be described by using conversation equations. If a model is described in
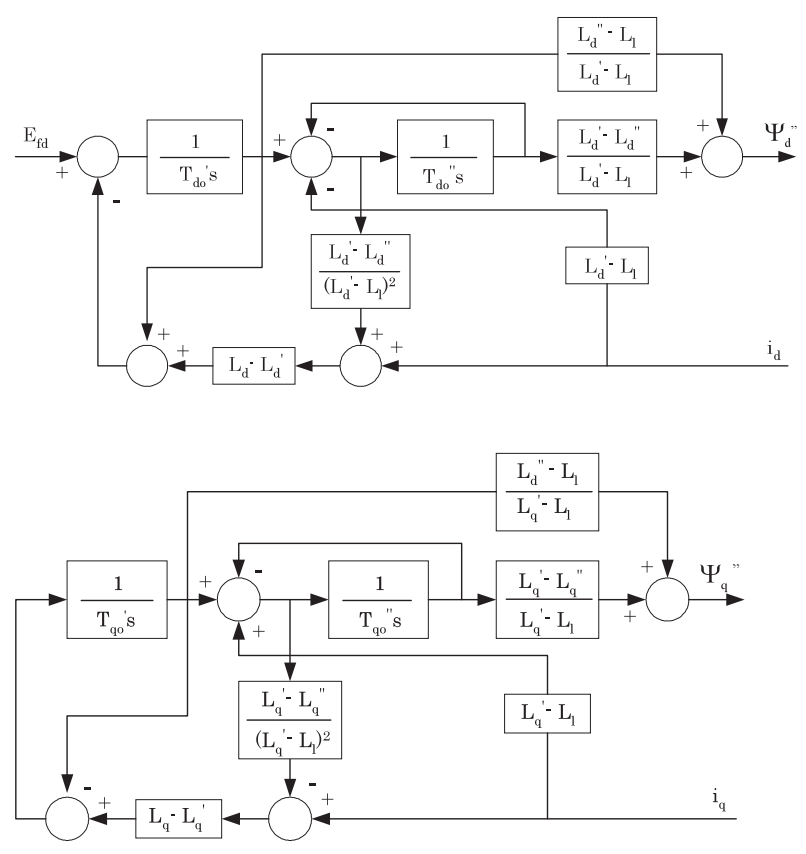

Fig. 8. Block diagram of flux-current relationship of the synchronous generator model GENROU in PSS/E

terms of quantities that are known and unknown, block diagram approach is often used. This is a way that we usually use when designing control systems. Modelica can combine both conversation equation and block diagram for modeling in the same model component. As an example, the Modelica coding of GENROU is shown as follows:

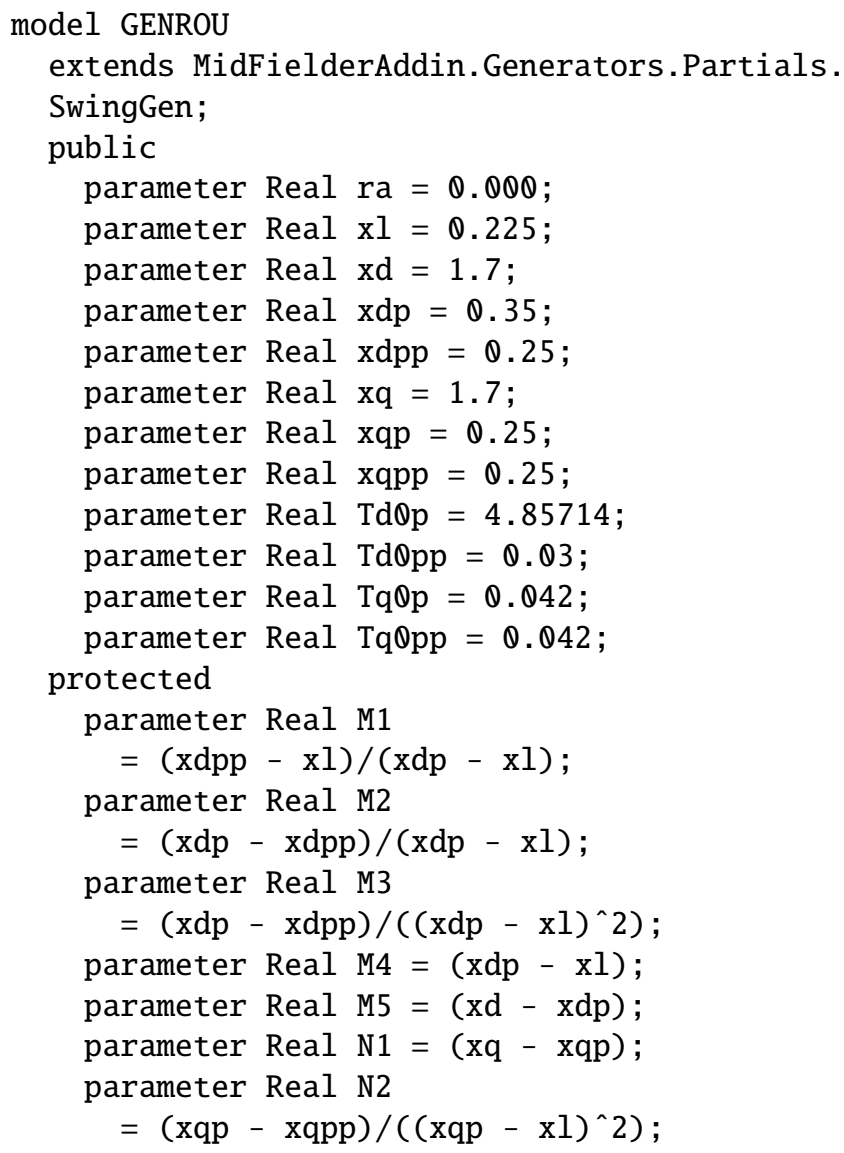




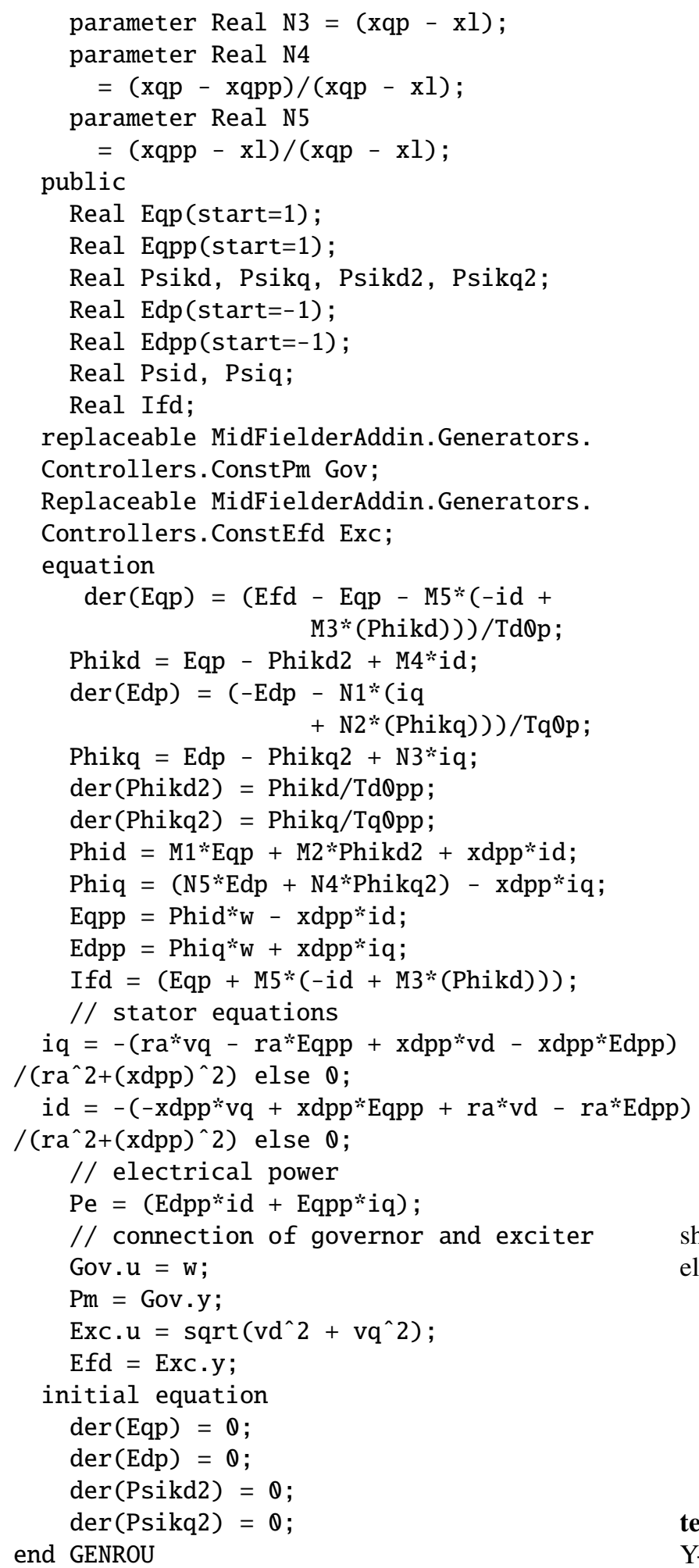

Here, the dynamic generator model inherits from SwingGen class which is a class that defines the swing equation and coordinate $\mathrm{d}-\mathrm{q}$ transformation. The equation in the model is derived from the block diagram in Fig. 8. Exciter and governor models have been declared using the modifier replaceable; they can be replaced for models taken from a user-defined model. For example, IEEJ LAT-1 type exciter/AVR model and IEEJ thermal and nuclear LPT-1 type turbine/governor model in Ref. (22) can be modeled using block diagrams as shown in Fig. 9 and Fig. 10, respectively. The following code

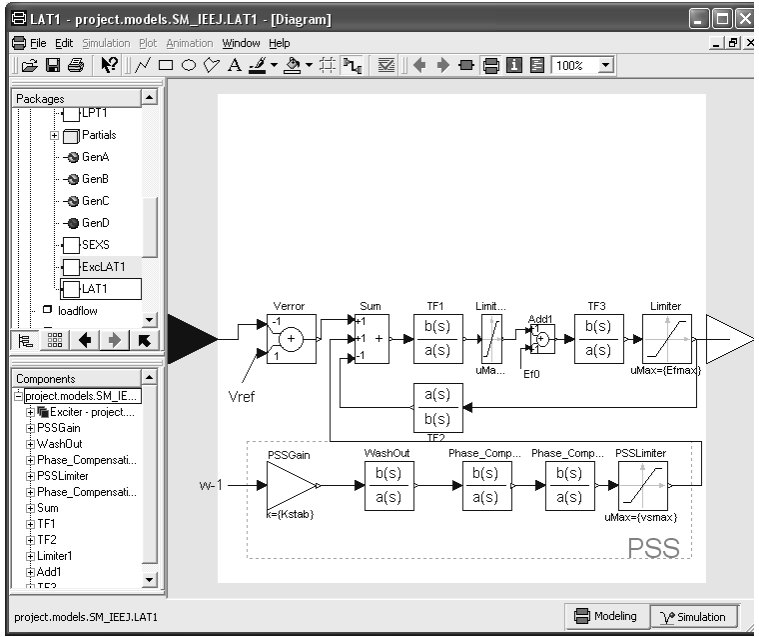

Fig. 9. Block diagram of LAT-1 type exciter/AVR model

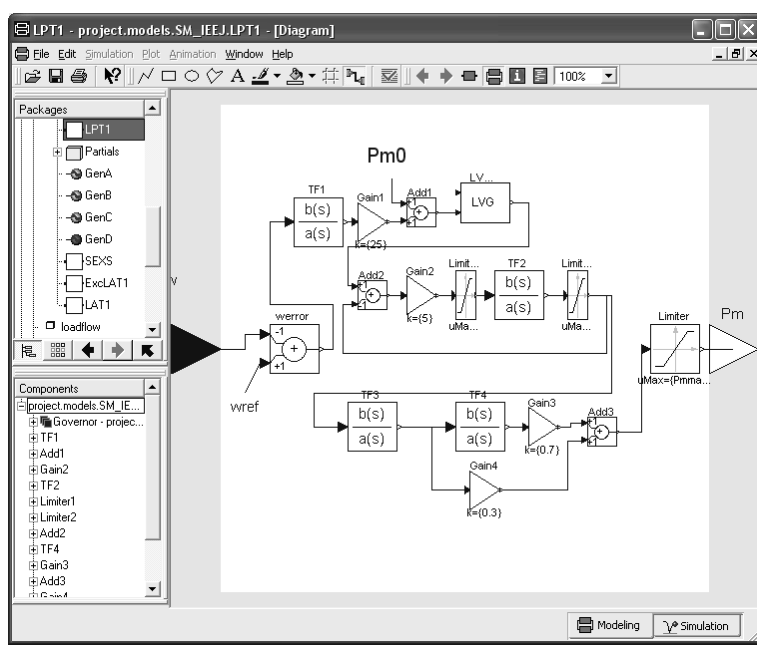

Fig. 10. Block diagram of IEEJ thermal and nuclear LPT-1 type turbine/governor model

shows how to replace user-defined exciter and governor models into a generator model.

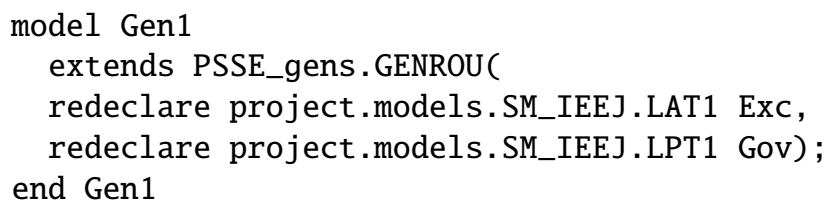

5.2 Study Case-IEEJ Standard Single Machine System Comparison results simulated by VTS in MidFielder, Y-method, PSS/E, MidFielderAddin (using D1Q2F1 generator in MidFielder) and MidFielderAddin (using GENROU generator in PSS/E) for one machine connected to infinite power system model as described in technical report of IEEJ are reported in this section. Study case no. 3 in the technical report $^{(22)}$ (a $3 \mathrm{LG}$ fault at the center of the transmission line, with re-closing action) is applied.

Study objective: Our primary objectives are to validate the accuracy of MidFielderAddin library and to test translators in data reading script layer.

Study method: Throughout this study case, the topology I as in Fig. 6 was used to carry out simulations. There is 
a constraint in using IEEJ exciter and governor models with PSS/E in this study because these models are non-standard models in the software package. PSS/E requires much effort to define a user-defined model that cannot be adequately simulated by a standard model. In order to simplify this study, simulations for two cases on IEEJ standard power system model were carried out. In the first case, we replaced IEEJ exciter and governor models with a simplified exciter (SEXS model in PSS/E ${ }^{(19)}$, and a constant power for governor system so as to enable the comparisons produced by the investigated simulation software packages. In the second case, IEEJ thermal and nuclear LPT-1 type turbine/governor model and IEEJ LAT-1 type exciter/AVR model were used. The result obtained from PSS/E was not included in Case 2 due to the above-mentioned constraint in PSS/E. In order to analyze the similarity of resulting waveforms, correlation index is calculated using 1 for 20-s period and is used to describe the degree to which one result is linearly related to another. If the two vectors are identical, the correlation inde $x$ is unity. Having obtained VTS in MidFielder as reference method, those methods having correlation index close to unity should give the result close to VTS in MidFielder.

$$
\text { correlation index }=\frac{\sum_{i=1}^{n} v_{1 i} v_{2 i}}{\sqrt{\sum_{i=1}^{n} v_{1 i}^{2} \sum_{i=1}^{n} v_{2 i}^{2}}}
$$

An analysis of system under fault condition is also necessary to verify the validity of the developed generator models for stability studies. The concept of critical clearing time (CCT) is used to define the stability limit of a generator. The $\mathrm{CCT}$ is defined as the maximum fault duration that a fault can remain on a power system network without losing the stability of machines. With the help of the simulation program, the CCT is evaluated for each simulation package. In this study, the investigation of CCT was carried out for Case 1.

Results and discussions: The results from two study cases are as follows:

[Case 1] With simplified exciter (SEXS: $\mathrm{TA}=0, \mathrm{~TB}=0.1$, $\mathrm{K}=10, \mathrm{TE}=0.1$, Efmax $=4, \mathrm{Efmin}=0$ ) and constant power for governor system.

The conclusion drawn from Table 1 is that all simulation programs provide results nearly identical. The different of the lowest index and the reference is about $0.0059 \%$. Therefore, we have strong believe in MidFielderAddin library that it can produce sufficiently accurate results. Figure 11 shows a comparison of simulation results using non-linear time domain simulation. We can see that there is a near perfect agreement among the results of all simulation programs.

[Case 2] With IEEJ LAT-1 type exciter/AVR and IEEJ thermal and nuclear LPT-1type turbine/governor.

Table 2 reveals that all methods provide results nearly identical. The different of the lowest index and the reference is about $0.0059 \%$. Figure 12 shows a comparison of simulation results using non-linear time domain simulation. We can see that there is also a near perfect agreement among the results of all simulation programs for Case 2 .

Here, we would like to give some remarks in this study. It
Table 1. Correlation index of Case 1

\begin{tabular}{|c|c|c|c|c|}
\hline VTS & Y-Method & PSS/E & $\begin{array}{c}\text { MidFielderAddin } \\
\text {-D1Q2F1 }\end{array}$ & $\begin{array}{c}\text { MidFielderAddin } \\
\text {-GENROU }\end{array}$ \\
\hline 1.00000 & 0.99994 & 0.99998 & 0.99996 & 0.99994 \\
\hline
\end{tabular}

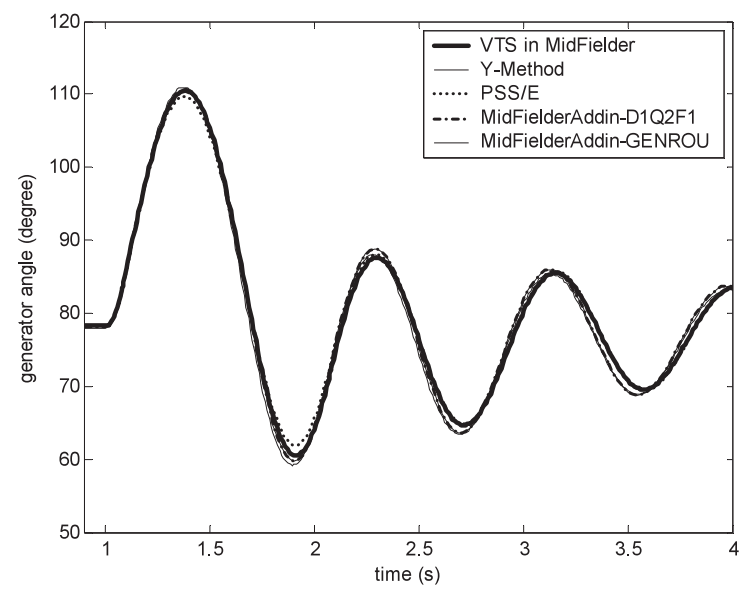

Fig. 11. Non-linear time domain simulation of Case 1

Table 2. Correlation index of Case 2

\begin{tabular}{|c|c|c|}
\hline VTS & Y-Method & MidFielderAddin-D1Q2F1 \\
\hline 1.00000 & 0.99994 & 0.99996 \\
\hline
\end{tabular}

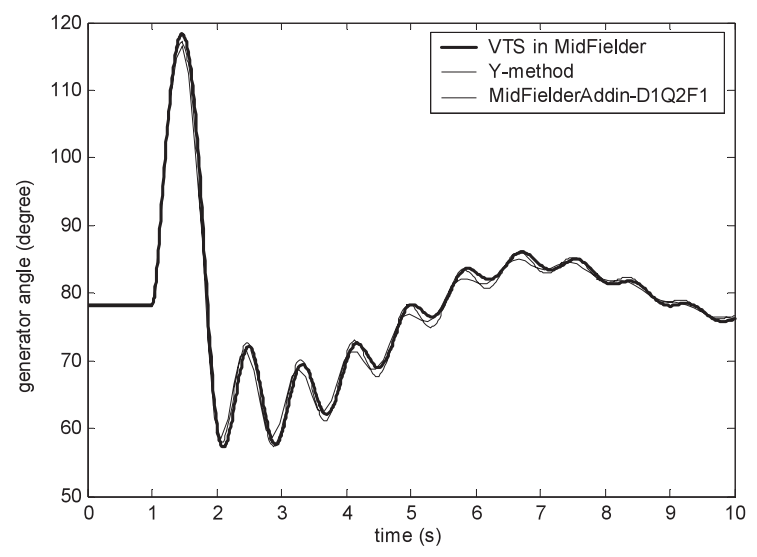

Fig. 12. Non linear time domain simulation of Case 2

should be noted that the main difference of generator models between MidFielder and PSS/E are:

- In PSS/E model, stator resistance $R_{a}$ is assumed to be zero whereas in MidFielder, the stator resistance can be taken into account.

- In PSS/E model, $X_{d}^{\prime \prime}$ must be equal to $X_{q}^{\prime \prime}$ whereas in MidFielder, the different values can be used.

Outside these assumptions, there is a possibility that PSS/E may not give results accurately whereas in MidFielder, these assumptions can be treated. As shown in the result figures of both cases, peak values, settle down time, final stable values, oscillation frequency and correlation index are sufficiently equal among the simulation packages. It can be concluded that our developed models in MidFielderAddin library have almost same accuracy as many commercial power 
system analysis packages. However, the conclusion using only a simple system may be not sufficient. In order to have stronger evidence to believe in the developed library, a study on much more complex network is done in the next section.

Table 3 shows the CCT calculation of case 1 for each simulation package. The values in the bracket show the level of differences in the calculated CCT when VTS is chosen as a

Table 3. CCT of each generator model and simulation package for single machine case

\begin{tabular}{|c|c|c|c|c|}
\hline $\begin{array}{c}\text { VTS } \\
(\mathrm{s})\end{array}$ & $\begin{array}{c}\text { Y-method } \\
(\mathrm{s})\end{array}$ & $\begin{array}{c}\text { PSS/E } \\
(\mathrm{s})\end{array}$ & $\begin{array}{c}\text { D1Q2F1 } \\
(\mathrm{s})\end{array}$ & $\begin{array}{c}\text { GENROU }^{*} \\
(\mathrm{~s})\end{array}$ \\
\hline 0.082 & 0.083 & 0.083 & 0.082 & 0.082 \\
$(0 \%)$ & $(1.22 \%)$ & $(1.22 \%)$ & $(0 \%)$ & $(0 \%)$ \\
\hline
\end{tabular}

Model in MidFielderAddin

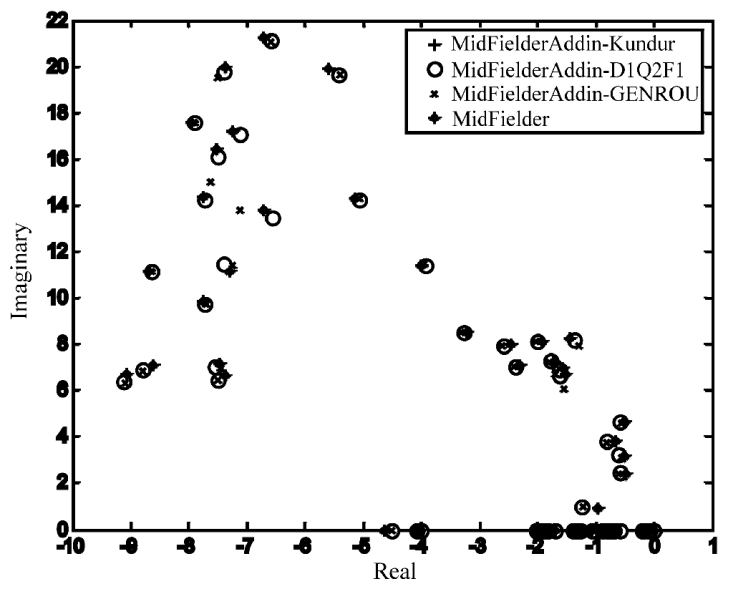

(a)

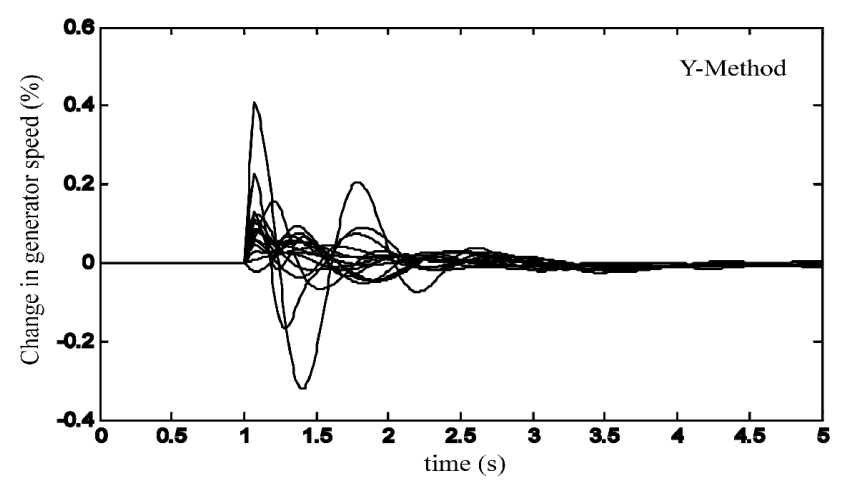

(c)

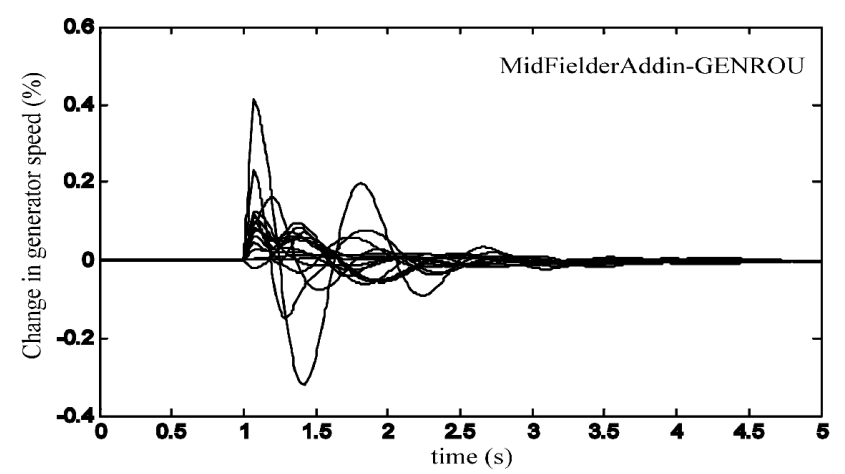

(e) base case. In overall, the results obtained are close enough to conclude that there is an agreement of models among software packages with few differences that are most likely to be caused by the numerical errors of each software package.

From these results, it can be concluded that MidFielderAddin library can provide compatibility with many commercial power system analysis packages. It provides very good results both in time domain simulation and stability limit estimation.

5.3 Study Case-16-Generator 68-Bus System The study system used in this section is a reduced order model of the New England/New York interconnection ${ }^{(23)}$. This system consists of 16 generators, 68 buses, 86 branches and 52 loads. We consider various types of detailed generator models including the dynamic behavior of exciters, power system stabilizers (PSSs) and governors as well as non-linear loads ${ }^{(23)(24)}$. With the help of the simulation program, the stability limit using CCT is evaluated and is used to confirm the validity of developed models.

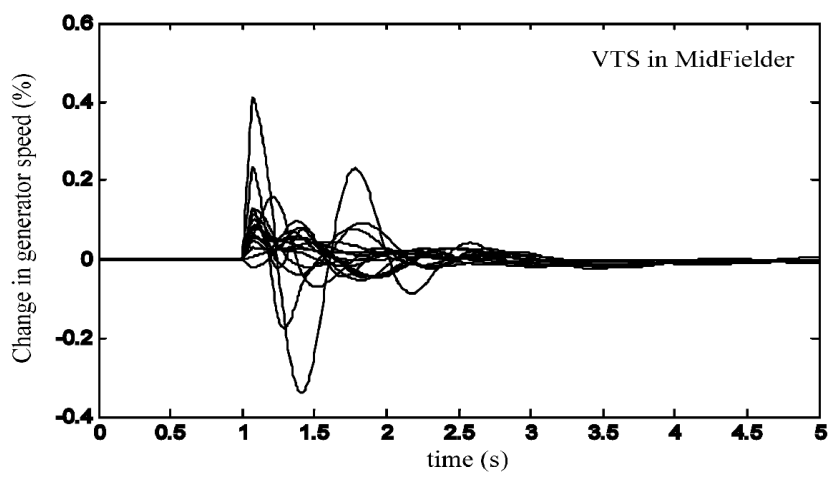

(b)

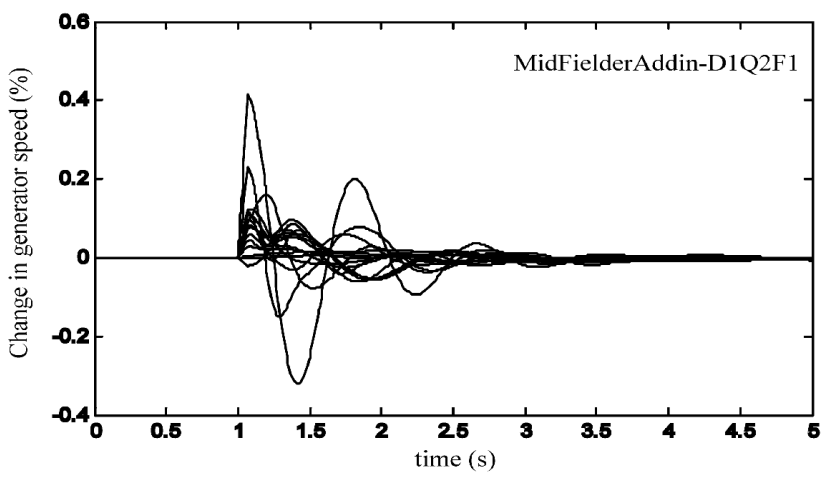

(d)

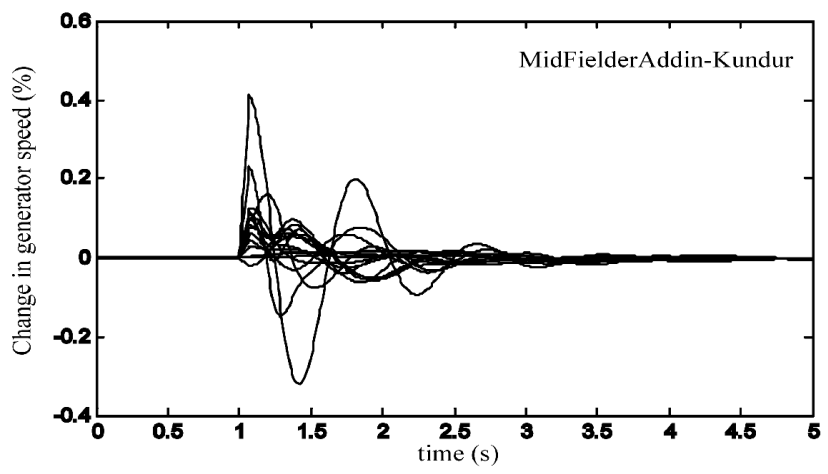

(f)

Fig. 13. Comparison results for 16-generator 68-bus system 
Study objective: The objectives of this study case are as follows:

- To validate the accuracy of MidFielderAddin library for multimachine case both in frequency and time domain.

- To show the ability of using object-oriented physical system modeling for a study on effects of generators from various types of models (This case is not easy for conventional power system simulation programs).

- To show the capability of Modelica for eigenvalues analysis study.

- To demonstrate post-processor ability for 3-D plot using the simulation result obtained from MidFielderAddin (This plot is useful for visualizing the power system).

Study method: The system data is taken from Ref. (23). PSSs are installed for all generators using same parameter setting as in Ref. (23). This system uses user-defined models for exciter and governor systems. The simulation events for this study are set up as follows:

- Three-phase to ground fault on Line 1-27 near Bus 1 of subsystem 3 at $\mathrm{t}=1$ second

- Clear fault and open Line 1-27 at $\mathrm{t}=1.07$ seconds

- Re-close Line 1-27 at t $=2.07$ seconds.

This fault occurs near area 3 on the tie line between area 1 and area 3. Considerable oscillatory activity should be obvious in the speed deviations because the fault occurs on the long and weak tie line which is one of the root causes of the low frequency inter-area oscillations. The topology II as in Fig. 7 was used to carry out simulations in this study case.

Results and discussions: After adding new models in Modelica, the program can calculate not only the non-linear time domain, but also eigenvalues for frequency domain analysis. In this study, the calculated eigenvalues obtained from Modelica were compared with those provided by eignevalues analysis tool in MidFielder. Eigenvalues analysis tool in MidFielder is an application to calculate eigenvalues precisely for large-scale power system by combining Arnoldi method and IIA method. It can treat large power system data which has 5,000 buses and 500 generators. We can see in Fig. 13(a) that the calculated eigenvalues by both programs are almost identical. In order to ensure that the compatibility of both programs is complete, we double checked the results in nonlinear time domain simulation. Here, we used VTS in MidFielder and Y-method as reference programs. The results in Fig. 13(b) to Fig. 13(f) show that there is a near perfect agreement among the results of both programs. Therefore, it can be concluded that MidFielderAddin library can provide perfect compatibility with MidFielder. Also, we show the results when the generator models are replaced by user-defined models such in Fig. 13(e) to Fig. 13(f). This case is not easy by many power system simulation programs, but it can be done automatically and easily with our proposed integration system. The results obtained in this section were carried out by using the Python script in MidFielder to automate processes in data reading, translating, application running and plotting. Therefore, users do not require to have special skills in software handling. Table 4 shows the calculated CCT of each type of generator models in MidFielderAddin and two simulation packages. The values in the bracket show the level of differences in the calculated CCT when VTS is chosen as a base case. The results obtained are close enough to conclude
Table 4. CCT of each generator model and simulation package for multimachine case

\begin{tabular}{|c|c|c|c|c|}
\hline $\begin{array}{c}\text { VTS } \\
(\mathrm{s})\end{array}$ & $\begin{array}{c}\text { Y-method } \\
(\mathrm{s})\end{array}$ & $\begin{array}{c}\text { D1Q2F1 } \\
(\mathrm{s})\end{array}$ & $\begin{array}{c}\text { GENROU }^{*} \\
(\mathrm{~s})\end{array}$ & $\begin{array}{c}\text { Kundur }^{*} \\
(\mathrm{~s})\end{array}$ \\
\hline 0.488 & 0.494 & 0.484 & 0.483 & 0.483 \\
$(0 \%)$ & $(1.23 \%)$ & $(-0.82 \%)$ & $(-1.02 \%)$ & $(-1.02 \%)$ \\
\hline
\end{tabular}

Model in MidFielderAddin

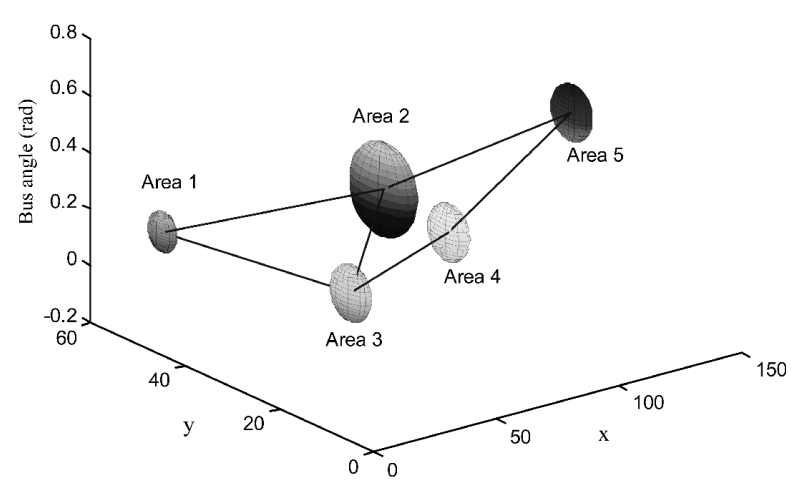

Fig. 14. 3-D plot at initial power flow of study 16generator 68-bus system

that there is an agreement of models among software packages with few differences that are most likely to be caused by the numerical errors of each software package. Additionally, we show the ability of post-processor in MidFielder to visualize a power system by using a 3-D plot. Figure 14 shows a 3-D plot of a study power system at initial power flow. At every snapshot, invoked by the timer settings, the image screen is captured and saved to a disk file. Therefore, we can make a 3-D animation easily.

\section{Conclusions}

MidFielder provides an easy-to-use high level user interface that works quite well for most application areas in power systems. However, for certain application areas that we may need new modeling, many power system analysis packages are not very intuitive and sometimes hard to access into the models and modify codes. Adaptability and flexibility is especially important for future integrated power system analysis packages, since they need to interact with a number of external tools, models and data formats, contain many different functions, and usually need to add new ones. Modeling environments need to be flexible to adapt to changing user needs. Without flexibility, power system analysis tools will become too hard to be used for practical needs, and may be stopped to be used. Our vision for the MidFielder integrated Modelica environment is to fulfill essentially all the requirements for a general purposes integrated power system analysis package combined with the specific needs for modeling and simulation environments. We believe that our purposed concept will have a great impact for the development of future integrated power system analysis package so that the integrated power system analysis package will have the completeness for industrial, educational and research purposes.

(Manuscript received March 8, 2006, revised Aug. 20, 2006) 


\section{References}

( 1 ) Modelica and the Modelica Association Web site: http://www.modelica.org/

( 2 ) M.M. Tiller: Introduction to Physical Modeling with Modelica, Kluwer Academic Publishers, Massachusetts (2001)

( 3 ) M. Larsson: "ObjectStab - a Modelica Library for Power System Stability Studies", Proc. of the 2000 Modelica Workshop, pp.13-22 (2000)

( 4 ) M. Larsson: "ObjectStab-An Educational Tool for Power System Stability Studies", IEEE Trans. Power Syst., Vol.19, No.1, pp.56-63 (2004)

( 5 ) K. Hongesombut, Y. Mitani, and K. Tsuji: "An Incorporated Use of Genetic Algorithm and a Modelica Library for Simultaneous Tuning of Power System Stabilizers", Proc. of $2^{\text {th }}$ International Modelica Conference, pp.89-98 (2002)

( 6 ) K. Hongesombut, Y. Mitani, and K. Tsuji: "Simultaneous Tuning of a Coordinated FACTS Device for Stability Enhancement Using a Micro-GA”, Proc. of the IASTED International Conference, pp.167-172 (2002)

( 7 ) K. Hongesombut, Y. Mitani, and K.Tsuji: "Optimal Location Assignment and Design of Superconducting Fault Current Limiters Applied to Loop Power Systems", IEEE Trans. Appl. superconduct., Vol.13, No.2, pp.18281831 (2003)

( 8 ) K. Hongesombut, Y. Mitani, and K.Tsuji: "Fuzzy Logic Controller Based on Observed Signals and a Genetic Algorithm Application with STATCOM for Power System Stabilization", IEEJ Trans. Power \& Energy, Vol.123, No.5, pp.589-596 (2003)

( 9 ) K. Hongesombut, Y. Mitani, and K.Tsuji: "Allocation and Design of Superconducting Fault Current Limiters in Loop Power Systems by a Genetic Algorithm", IEEJ Trans. Power \& Energy, Vol.123, No.9, pp.1054-1063 (2003)

(10) S. Dechanupaprittha, et al.: "Design of Robust SMES Controller in a Multimachine Power System By Using Hybrid TS/EP", Proc. of $15^{\text {th }}$ Power Systems Computation Conferences, in CD-ROM (2005)

(11) Y. Tada, et al.: "Development of an Integrated Power System Analysis Package", IEEE PES. PowerCON, pp.1695-1700 (2000)

(12) Y. Tada, et al.: "A BCU-DSA System for On-line Dynamic Security Assessments of Large-scale Power Systems", Proc. of $14^{\text {th }}$ Power System Computation Conference, in CD-ROM (2002)

(13) Y. Tada, et al.: "Development of Voltage Stability Constrained OPF as one of the functions of the Integrated Power System Analysis Package, named IMPACT", International Power Engineering Conferernce, in CD-ROM (2003)

(14) Y. Tada, et al.: "Enhancement of Data Hndling In Integrated Power System Analysis Package, Named IMPACT, Using Information Technologies", IFAC Symposium on Power Plants \& Power Systems Control, pp.823-828 (2003)

(15) Dymola Web site: http://dynasim.com/

(16) MathModelica Web site: http://www.mathcore.com/

(17) Open Modelica Project Web site: http://www.ida.liu.se/ppelab/modelica/

(18) H. Elmqvist, et al.: Dymola—User's Manual, DynaSim AB, Lund (2002)

(19) PSS/E-29 On-line Documentation, Power Technologies, Inc., Schenectady (2002)

(20) K. Hongesombut, et al.: "Object-Oriented Modeling for Advanced Power System Simulations", Proc. of 2005 IEEE St. Peterburg PowerTech, in CDROM (2005)

(21) K. Hongesombut, et al.: "Integration of Modelica Data Conversion Function in Integrated Power System Analysis Package, named IMPACT", Proc. of the $15^{\text {th }}$ Annual Conference of Power \& Energy Society, IEE of Japan (2004)

(22) IEEJ Electrical Power System Standard Models, Technical Report, No.754 (1999)

(23) G.J. Rogers: Power System Oscillations, Kluwer Academic Publishers, Boston (2000)

(24) P. Kundur: Power System Stability and Control, McGrawHill, New York (1993)
Komsan Hongesombut (Member) obtained B.Eng, First Class

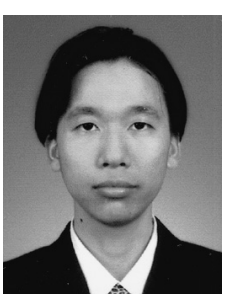
Honours degree in Electrical Engineering and M.Eng. degree in Electrical Engineering from King Mongkut's Institute of Technology Ladkrabang, Thailand, in 1997 and 1999, respectively. In 2003, he obtained Ph.D. in Electrical Engineering from Osaka University, Japan. From 2003 to 2005, he was a postdoctoral fellowship, funded by the Japan Society for the Promotion of Science (JSPS), at the Department of Electrical Engineering of Kyushu Institute of Technology. Currently, he is a visiting researcher of the Power System Technology Group at the Electric Power Engineering R\&D Center of Tokyo Electric Power Company.

Tsuyoshi Takazawa (Member) received a B.S. degree from Waseda

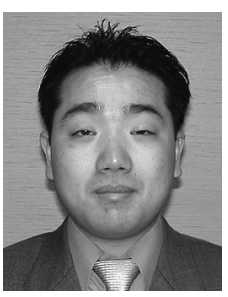
University in 1997. He joined Tokyo Electric Power Company in 1997, where he is a research staff member of the Power System Technology Group at the Electric Power Engineering R\&D Center of Tokyo Electric Power Company.

Yasuyuki Tada (Member) joined Tokyo Electric Power Company after graduating from Kawagoe Technical High School, Saitama Prefecture in 1981. After graduating from TEPCO Education Institute in 1988, he has engaged in the Power System Technology Group, R\&D Center of Tokyo Electric Power Company. From 1989 to 1990 he was a visiting researcher at the University of Tokyo. He received Dr. Eng. Degree from the University of Tokyo in 1998.

Yasunori Mitani (Member) received B.Sc., M.Sc. and D.Eng. de-

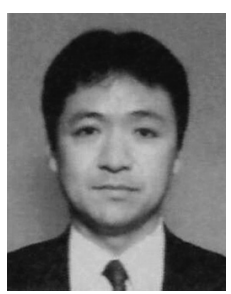
(Member) received B.Sc., M.Sc. and D.Eng. de-
grees in Electrical Engineering from Osaka University, Japan in 1981, 1983 and 1986, respectively. He was a Visiting Research Associate at the University of California, Berkeley, from 1994 to 1995. He is currently a professor at the Department of Electrical Engineering, Kyushu Institute of Technology. His research interests are in the areas of analysis and control of power systems. He is a member of the Institute of Electrical Engineers of Japan and IEEE. 